bacteraemia were 6.5 times more likely to die in hospital than to be discharged alive (adj-OR=6.50, 95\%CI 5.95-7.11). The most common organisms identified were Escherichia coli and Staphylococcus aureus, present in $22.9 \%$ and $18.1 \%$ respectively, of the 2,265 admissions with a positive blood culture. The prevalence of MDR bacteria was low (5.6\%).

Conclusions Morbidity and mortality related to sepsis/bacteraemia in patients with cirrhosis remains a critical clinical problem.

\section{PWE-46 IMPACT OF THE COVID-19 PANDEMIC ON PATIENTS WITH HEPATOCELLULAR CARCINOMA IN THE WEST OF SCOTLAND}

${ }^{1}$ Alistair Mclaren*, ${ }^{2}$ Johannes Spoor, ${ }^{1}$ Gregory Naylor, ${ }^{1}$ Douglas Cartwright, ${ }^{2}$ Adrian J Stanley, 'Thomas Ronald Jeffrey Evans. 'Beatson West of Scotland Cancer Centre, Glasgow, UK; ${ }^{2}$ Glasgow Royal Infirmary, Glasgow, UK

\subsection{6/gutjnl-2021-BSG.236}

Introduction The COVID-19 pandemic has focused medical resources on acute and critical care, with an impact on patients with chronic illness. Worldwide, HCC surveillance, imaging and clinic follow-up may have been impacted, which is likely to worsen outcomes. In the West of Scotland, all patients with HCC are discussed at our weekly regional MDT. We examined the difference in outcomes for new patients referred to this MDT and patients returning to the MDT to see if there were differences during the COVID-19 pandemic compared to the previous year.

Methods We collected data on patients reviewed in the West of Scotland HCC MDT between April - October 2020; compared to the same period in 2019. Statistical analysis was performed using ' $\mathrm{R}$ ' ( $\mathrm{R}$ Foundation for Statistical Computing, Vienna, Austria).

Results There were 128 patients discussed in April - October 2020: 69 patients presenting to the MDT for the first time ('new') and 59 patients that had been discussed previously ('return') compared to 155 (93 new) in April - October 2019. Amongst new patients discussed, median overall survival was not reached, but 12-month survival rate was 30\% (95\% CI $0.13-0.70)$ in 2020 compared to $54 \%$ in 2019 (95\%CI $0.44-0.64, p=0.10)$. For return and new patients combined, the median overall survival was 11.3 months in 2020 (95\% CI 6.93 - not reached) vs 16.9 months in 2019 (95\% CI 13.8 - not reached), and 1 year survival rate was $48 \%$ in 2020 (95\% CI $0.38-0.62$ ) compared to $62 \%$ in 2019 (95\%CI $0.55-0.70)$. As seen in figure 1 , there was a significant decrease in survival in combined new and return patients with HCC presented to the MDT in 2020 compared to 2019 ( $p=$ 0.04) There was no significant difference in median Child Pugh score (A6 in both 2020 and 2019), median ECOG performance status (1 in both 2020 and 2019), median AFP $(12.0 \mathrm{kU} / \mathrm{L}$ in $2020,7.0 \mathrm{kU} / \mathrm{L}$ in $2019 p=0.28)$, and median diameter of largest lesion $(4.1 \mathrm{~cm}$ in $2020,3.0 \mathrm{~cm}$ in 2019 $p=0.17)$. A higher proportion of patients were referred for TACE (20\% vs 15\%), and Best Supportive Care (43\% vs $32 \%)$ in 2020 compared to 2019 , with a lower proportion being referred for RFA (11\% vs 15\%) and a similar proportion for SACT (11\%) and surgery (3\%).

Conclusions We identified significantly reduced survival in patients with HCC assessed at our MDT during the COVID19 pandemic compared with the same period in 2019. This is despite no significant difference in factors that influence survival. This may continue to have implications continuing during recovery from the pandemic and requires further study.

\section{PWE-47 CLINICAL PRESENTATION AND OUTCOMES OF WILSON'S DISEASE: A SINGLE CENTRE COHORT STUDY}

Akhilesh Mulay*, James Dooley, Emmanouil Tsochatzis. UCL Institute for Liver and Digestive Health, Royal Free Hospital and UCL, London, UK

\subsection{6/gutjnl-2021-BSG.237}

Introduction Wilson's Disease is a disorder of copper homeostasis leading to hepatic and neuropsychiatric disease. Clinical data on these patients are limited due to low disease frequency. This report explores the presentation, management and outcomes of a large cohort of patients with Wilson's disease including pregnancy outcomes.

Methods A retrospective analysis was performed on 63 patients with Wilson's disease managed at the Royal Free Hospital, London, between 2012 and 2021. Clinical data were collected on presenting features, investigations, treatment and outcomes.

Abstract PWE-46 Figure 1 Overall Survival of all patients with HCC discussed in the MDT in 2019 (Pre COVID) vs 2020 (During COVID) 